\title{
Routine Screening for KIT M541L is Not Warranted in the Diagnostic Work-up of Patients with Hypereosinophilia
}

\author{
Yvette Hoade ${ }^{1,2}$, Georgia Metzgeroth ${ }^{3,4}$, Juliana Schwaab ${ }^{3,4}$, \\ Andreas Reiter ${ }^{3,4}$, Nicholas C. P. Cross ${ }^{1,2}$,
}

1. Wessex Regional Genetics Laboratory, Salisbury, UK

2. Faculty of Medicine, University of Southampton, Southampton, UK

3. Department of Hematology and Oncology, University Medical Centre Mannheim, Mannheim, Germany

4. Medical Faculty Mannheim, Heidelberg University, Heidelberg, Germany

Abbreviated Title: KIT M514L in hypereosinophilia

Key Terms: KIT, M541L, eosinophilia, hypereosinophilia, HE, hypereosinophilic syndrome, HES

Word Count: 741

Numbers of Figures and Tables: 2

\section{Address for Correspondence:}

Nicholas C.P. Cross

Professor of Human Genetics

Salisbury District Hospital

Salisbury,

SP2 8BJ

UK

Tel: +(44) 1722429080

Fax: + (44) 1722331531

email: ncpc@soton.ac.uk 
Hypereosinophilia (HE), defined as peripheral blood eosinophil counts persistently $>1.5$ $x 10^{9} / L$, [1] is seen in a wide range of reactive and clonal disorders. In hypereosinophilic syndrome (HES), HE results in potentially life-threatening organ damage as a consequence of eosinophilic tissue infiltration and/or release of toxic granule proteins [2]. The underlying causes of HE are complex, heterogeneous and incompletely understood, but an important subset is characterized by clonal HE driven by an underlying acute or chronic myeloid or lymphoid neoplasms [2] [3].

Clonal HE is frequently associated with somatic mutations that result in the constitutive activation of specific tyrosine kinases (TK), in particular PDGFR $\alpha$, PDGFR $\beta$, FGFR1, ABL, JAK2 or FLT3 [2]. Most commonly, translocations or other genomic rearrangements generate TK fusion genes, e.g. FIP1L1-PDGFRA, ETV6-PDGFRB, ZMYM2-FGFR1, PCM1-JAK2 and many others [2], but some cases test positive for activating TK point mutations such as KIT D816V or JAK2 V617F [4]. Identifying driver fusion genes is critical for clinical management, for example patients with PDGFRA or PDGFRB rearrangements show excellent responses to imatinib, with most cases achieving sustained haematological and molecular remissions $[5,6]$ [7]. Clinical benefit has also been achieved with TK inhibitors (TKI) for other abnormalities, for example ruxolitinib and sorafinib for cases with JAK2 or FLT3 fusions, respectively $[8,9]$.

Some cases of HE show clinical responses to imatinib despite no evidence for a recognized underlying abnormality in an imatinib-sensitive fusion gene [10]. For many of these cases responses are transient [5] but occasional longer term responders have been reported [7]. Recently it has been suggested that a somatically-acquired KIT variant c.1621 A>C; p.Met541Leu (hereafter referred to as KIT M541L) is associated with imatinib response in HE patients negative for PDGFRA/B rearrangements [11]. Whilst this finding is supported by the fact that M541L has been reported to increase the sensitivity of the KIT receptor to stem cell factor [12], it remains controversial because this variant is a recognised inherited single nucleotide polymorphism ( $r$ 3822214) with a minor allele frequency of 0.08 in the ExAC database (http://exac.broadinstitute.org/) and is classified as benign/likely benign on ClinVar (www.ncbi.nlm.nih.gov/clinvar/). Nevertheless, it has been suggested that HES patients should be screened for KIT M541L, as positive cases may benefit from imatinib treatment [11]. 
To evaluate the significance of KIT M514L in $\mathrm{HE}$, we aimed to (i) compare the KIT M541L allele frequency between patients referred for investigation of HE and normal healthy controls (ii) investigate the variant allele frequency (VAF) in positive HE cases to determine if KIT M541L mutations may be acquired somatically and (iii) investigate the KIT M514L status in cases negative for PDGFRA/B rearrangements who responded to imatinib.

We screened healthy controls $(n=214)$ and patients referred for investigation of FIP1L1PDGFRA negative HE ( $\mathrm{n}=220)$ for KIT M541L using an amplification refractory mutation system (ARMS) PCR designed to amplify allele specific products of different sizes, and able to detect KIT M541L down to 5\% VAF (Figure 1a). Forty two (19\%) of HE cases tested positive for KIT M541L compared to $38(18 \%)$ of healthy controls. The allele frequency between the control and HE groups was indistinguishable (0.095 versus $0.098 ; \mathrm{P}=0.91$; Fishers exact two tailed test). Digital droplet PCR (ddPCR) was used for patients heterozygous for KIT M541L by the ARMS assay to determine whether the variant burden was close to $50 \%$ (consistent with a constitutional polymorphism) or significantly $<50 \%$ (suggestive of a somatic mutation). Of the $42 \mathrm{KIT} \mathrm{M541L} \mathrm{heterozygous} \mathrm{HE} \mathrm{cases,} 40$ had sufficient DNA for analysis. The median allele burden was $50.2 \%$ (range $47.9 \%-56.0 \%$ ), indistinguishable from that need in healthy controls $(n=28)$ and thus consistent with all instance of KIT M514L in HE being constitutional (Figure 2).

Finally, we studied pre-treatment DNA from 3 patients with HES negative for PDGFRA/B rearrangements who were treated with imatinib (400 mg/day) and showed normalization of eosinophil counts at a median of 0.8 months (range, $0.4-5.0$ ) after treatment for a duration of 13.6 months (range, 3.7-44.8). None of the three imatinib responders tested positive for KIT M541L prior to treatment (Figure 1b).

In conclusion, (i) we found no increased prevalence of KIT M541L in HE patients compared to controls, (ii) no case where KIT M514L was acquired somatically, and (iii) this variant was not present in three HES patients who responded to imatinib. Whilst we cannot exclude the possibility that KIT M541L may be acquired somatically in very rare cases, we conclude that 
there is no clinical value in screening for this variant on a routine basis in patients with $\mathrm{HE}$ or HES. 
A

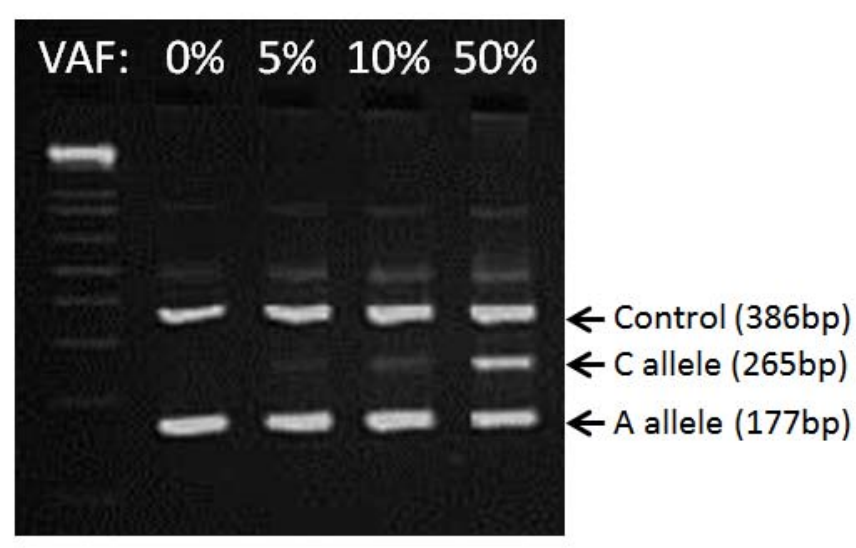

B

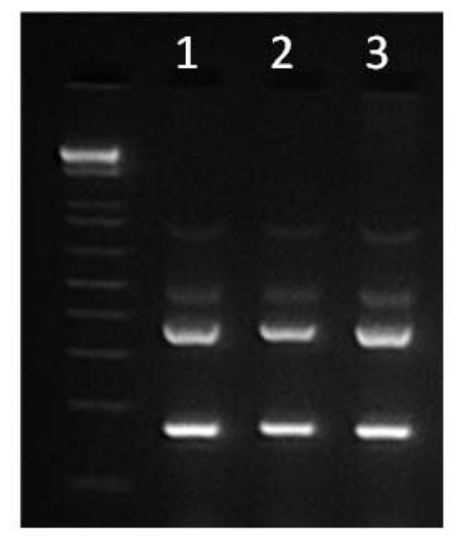

Figure 1: ARMS PCR to detect KIT M541L. (A) Concentration gradient demonstrating detection of KIT M541L at different \%VAF. (B) Absence of KIT M541L ARMS in three PDGFRA/B negative HES patients who responded to imatinib. A tetra-primer ARMS assay was designed using http://primer1.soton.ac.uk/primer1.html with inner primers designed to specifically amplify the normal and mutant KIT M541L sequences and outer primers to produce a positive control band for each reaction. Inner primers included mismatches to maximise allele discrimination (shown in lower case) and wild type/mutant specific bases (underlined). PCR primers were: forward outer (FO), 5'-

GTACAATGTAACCAAGGTGAAGCTCTGA-3'; reverse outer (RO), 5'-

ACAACCTTCCACTGTACTTCATACATGG-3'; forward wild-type-specific (Fwt), 5'-

TAGCTGGCATGATGTGCATTATTGcGA-3'; reverse mutant specific (Rmt), 5'ACCTGTAAATATTTGTAGGTCAGAATtAG -3 '. FO, RO and Rwt primers were all used at a final concentration of $0.5 \mu \mathrm{M}$ and Rmt was used at $1 \mu \mathrm{M}$ in PCR reactions using AmpliTaq Gold DNA polymerase, $25 \mathrm{ng}$ genomic DNA an annealing temperature of $60^{\circ} \mathrm{C}$, and 35 cycles. Each product was a different size that could be clearly resolved on a $3 \%$ agarose gel stained with ethidium bromide. 


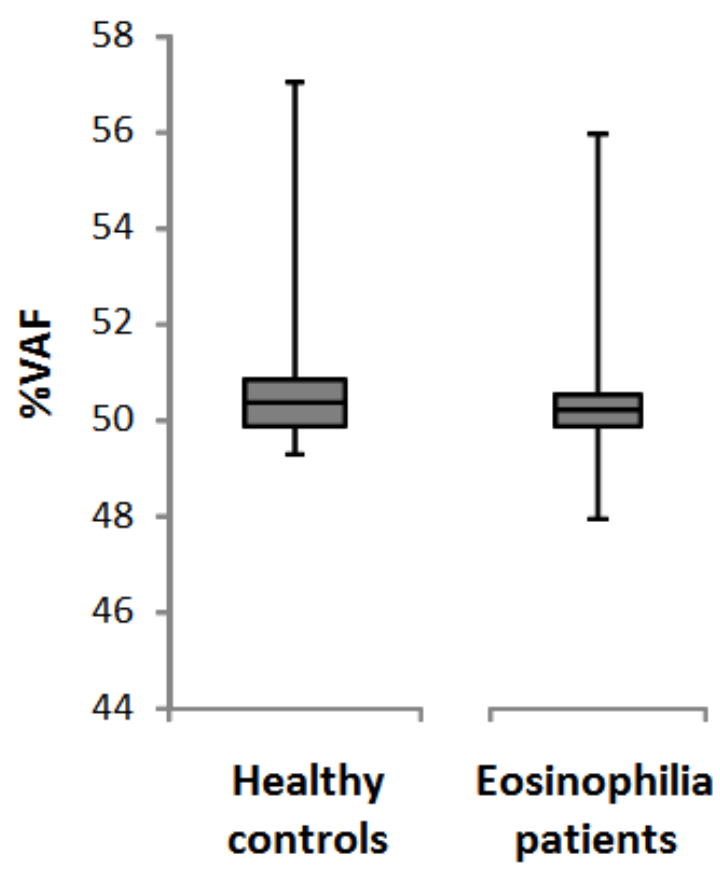

Figure 2: VAF for KIT M541L positive samples $(n=40)$ and heterozygous healthy controls $(n=28)$ tested by ddPCR. Mutant and wild-type specific probes were designed with 5' 6FAM and HEK reporters respectively and 3' BHQ1 (black hole quencher) and were used in the same reaction as forward and reverse primers. Probes and primers were: wild-type probe, 5'-[HEX]TGCATTATTGTGATGATTCTGACCTAC[BHQ1]-3'; mutant probe, 5'-[HEX] TGCATTATTGTGCTGATTCTGACCTAC[BHQ1]-3'; forward primer, 5'-TGCTGATTGGTTTCGTAA3'; reverse primer, 5'-CCTTCCACTGTACTTCATA-3'. Reactions were prepared with $117 \mathrm{ng}$ sonicated genomic DNA, $0.25 \mu \mathrm{M}$ of each probe, $0.9 \mu \mathrm{M}$ of each primer and 'no dUTP' Supermix (BioRad, Watford, UK). Droplets were prepared using QX200 Droplet Generator (BioRad). Amplification was performed with an annealing temperature of $58^{\circ} \mathrm{C}$ for $40 \mathrm{cycles}$ and droplets were measured using QX200 Droplet Reader (Biorad). Each sample was run in triplicate, with KIT M541L positive, negative and no template controls for each run. \%VAF was calculated by dividing the concentration of KIT M541L mutant droplets by the total concentration and multiplying by 100 . Only samples with an accepted droplet number of over 10000 were included as lower droplet numbers may produce less reliable results. Plots show the median, range and interquartile range. 


\section{References}

1 Valent P, Klion AD, Horny HP, Roufosse F, Gotlib J, Weller PF, Hellmann A, Metzgeroth G, Leiferman KM, Arock M, Butterfield JH, Sperr WR, Sotlar K, Vandenberghe P, Haferlach T, Simon HU, Reiter A, Gleich GJ: Contemporary consensus proposal on criteria and classification of eosinophilic disorders and related syndromes. The Journal of allergy and clinical immunology 2012;130:607-612 e609.

2 Reiter A, Gotlib J: Myeloid neoplasms with eosinophilia. Blood 2017;129:704-714.

3 Butt NM, Lambert J, Ali S, Beer PA, Cross NC, Duncombe A, Ewing J, Harrison CN, Knapper S, McLornan D, Mead AJ, Radia D, Bain BJ, British Committee for Standards in H: Guideline for the investigation and management of eosinophilia. British journal of haematology 2017;176:553-572.

4 Schwaab J, Umbach R, Metzgeroth G, Naumann N, Jawhar M, Sotlar K, Horny HP, Gaiser T, Hofmann WK, Schnittger S, Cross NC, Fabarius A, Reiter A: KIT D816V and JAK2 V617F mutations are seen recurrently in hypereosinophilia of unknown significance. American journal of hematology 2015;90:774-777.

5 Baccarani M, Cilloni D, Rondoni M, Ottaviani E, Messa F, Merante S, Tiribelli M, Buccisano F, Testoni N, Gottardi E, de Vivo A, Giugliano E, lacobucci I, Paolini S, Soverini S, Rosti G, Rancati F, Astolfi C, Pane F, Saglio G, Martinelli G: The efficacy of imatinib mesylate in patients with FIP1L1PDGFRalpha-positive hypereosinophilic syndrome. Results of a multicenter prospective study. Haematologica 2007;92:1173-1179.

6 Cheah CY, Burbury K, Apperley JF, Huguet F, Pitini V, Gardembas M, Ross DM, Forrest D, Genet P, Rousselot P, Patton N, Smith G, Dunbar CE, Ito S, Aguiar RC, Odenike O, Gimelfarb A, Cross NC, Seymour JF: Patients with myeloid malignancies bearing PDGFRB fusion genes achieve durable long-term remissions with imatinib. Blood 2014;123:3574-3577.

7 Metzgeroth G, Walz C, Erben P, Popp H, Schmitt-Graeff A, Haferlach C, Fabarius A, Schnittger S, Grimwade D, Cross NC, Hehlmann R, Hochhaus A, Reiter A: Safety and efficacy of imatinib in chronic eosinophilic leukaemia and hypereosinophilic syndrome: a phase-II study. British journal of haematology 2008;143:707-715.

8 Schwaab J, Knut M, Haferlach C, Metzgeroth G, Horny HP, Chase A, Tapper W, Score J, Waghorn K, Naumann N, Jawhar M, Fabarius A, Hofmann WK, Cross NC, Reiter A: Limited duration of complete remission on ruxolitinib in myeloid neoplasms with PCM1-JAK2 and BCR-JAK2 fusion genes. Annals of hematology 2015;94:233-238.

9 Jawhar M, Naumann N, Knut M, Score J, Ghazzawi M, Schneider B, Kreuzer KA, Hallek M, Drexler HG, Chacko J, Wallis L, Fabarius A, Metzgeroth G, Hofmann WK, Chase A, Tapper W, Reiter A, 
Cross NCP: Cytogenetically cryptic ZMYM2-FLT3 and DIAPH1-PDGFRB gene fusions in myeloid neoplasms with eosinophilia. Leukemia 2017;31:2271-2273.

10 Cools J, DeAngelo DJ, Gotlib J, Stover EH, Legare RD, Cortes J, Kutok J, Clark J, Galinsky I, Griffin JD, Cross NC, Tefferi A, Malone J, Alam R, Schrier SL, Schmid J, Rose M, Vandenberghe P, Verhoef G, Boogaerts M, Wlodarska I, Kantarjian H, Marynen P, Coutre SE, Stone R, Gilliland DG: A tyrosine kinase created by fusion of the PDGFRA and FIP1L1 genes as a therapeutic target of imatinib in idiopathic hypereosinophilic syndrome. The New England journal of medicine 2003;348:12011214.

11 Iurlo A, Gianelli U, Beghini A, Spinelli O, Orofino N, Lazzaroni F, Cambiaghi S, Intermesoli T, Rambaldi A, Cortelezzi A: Identification of kit(M541L) somatic mutation in chronic eosinophilic leukemia, not otherwise specified and its implication in low-dose imatinib response. Oncotarget 2014;5:4665-4670.

12 Foster R, Byrnes E, Meldrum C, Griffith R, Ross G, Upjohn E, Braue A, Scott R, Varigos G, Ferrao P, Ashman LK: Association of paediatric mastocytosis with a polymorphism resulting in an amino acid substitution (M541L) in the transmembrane domain of c-KIT. The British journal of dermatology 2008;159:1160-1169. 